\title{
SILICATE DUST IN THE ENVIRONMENT OF RS OPHIUCHI FOLLOWING THE 2006 ERUPTION
}

\author{
A. Evans, ${ }^{1}$ C. E. Woodward, ${ }^{2}$ L. A. Helton, ${ }^{2}$ J. Th. van Loon, ${ }^{1}$ R. K. Barry, ${ }^{3}$ M. F. Bode, ${ }^{4}$ R. J. Davis, ${ }^{5}$ J. J. Drake, ${ }^{6}$ \\ S. P. S. Eyres, ${ }^{7}$ T. R. Geballe, ${ }^{8}$ R. D. Gehrz, ${ }^{2}$ T. Kerr, ${ }^{9}$ J. Krautter, ${ }^{10}$ D. K. Lynch, ${ }^{11}$ J.-U. Ness, ${ }^{12}$ T. J. O’Brien, ${ }^{5}$ \\ J. P. Osborne, ${ }^{13}$ K. L. Page, ${ }^{13}$ R. J. Rudy, ${ }^{11}$ R. W. Russell, ${ }^{11}$ G. Schwarz, ${ }^{14}$ S. Starrfield, ${ }^{12}$ And V. H. Tyne ${ }^{1}$ \\ Received 2007 September 17; accepted 2007 October 26; published 2007 November 14
}

\begin{abstract}
We present further Spitzer Space Telescope observations of the recurrent nova RS Ophiuchi, obtained over the period 208-430 days after the 2006 eruption. The later Spitzer IRS data show that the line emission and free-free continuum emission reported earlier is declining, revealing incontrovertible evidence for the presence of silicate emission features at 9.7 and $18 \mu \mathrm{m}$. We conclude that the silicate dust survives the hard radiation impulse and shock blast wave from the eruption. The existence of the extant dust may have significant implications for understanding the propagation of shocks through the red giant wind and likely wind geometry.
\end{abstract}

Subject headings: binaries: close — binaries: symbiotic — infrared: stars — novae, cataclysmic variables — stars: individual (RS Oph)

\section{INTRODUCTION}

RS Oph is a recurrent nova (RN) that erupted in 1898, 1933, 1958, 1967, and 1985 (Wallerstein 2008). It consists of a semidetached binary (orbital period 455.7 days) comprising a Roche-lobe-filling red giant (M2 III) and a massive $\left(\gtrsim 1.2 M_{\odot}\right)$ white dwarf (WD; Fekel et al. 2000). The eruption follows a thermonuclear runaway on the surface of the WD (Starrfield et al. 1988). In the case of the RS Oph class of RNs, however, the ejected material runs into, and shocks, a dense red giant (RG) wind (Bode \& Kahn 1985; O'Brien et al. 1992).

The 1985 eruption was, for the first time, the subject of a multiwavelength observational campaign, from the radio to the X-ray (Bode 1987). Its most recent eruption, on 2006 February 12.83 (Narumi et al. 2006; we take this to define the origin of time post-outburst), was the subject of an even more intensive

\footnotetext{
${ }^{1}$ Astrophysics Group, Lennard-Jones Laboratories, Keele University, Staffordshire ST5 5BG, UK; ae@astro.keele.ac.uk, jacco@astro.keele.ac.uk, vht@astro.keele.ac.uk.

${ }^{2}$ Department of Astronomy, School of Physics and Astronomy, 116 Church Street SE, University of Minnesota, Minneapolis, MN 55455; ahelton@ astro.umn.edu, chelsea@astro.umn.edu, gehrz@astro.umn.edu.

${ }^{3}$ Exoplanets and Stellar Astrophysics Laboratory, NASA Goddard Space Flight Center, Greenbelt, MD 20771; richard.k.barry@nasa.gov.

${ }^{4}$ Astrophysics Research Institute, Liverpool John Moores University, Twelve Quays House, Birkenhead CH41 1LD, UK; mfb@astro.livjm.ac.uk.

${ }^{5}$ Jodrell Bank Centre for Astrophysics, University of Manchester, M13 9PL, UK; tob@jb.man.ac.uk, rjd@jb.man.ac.uk.

${ }^{6}$ Harvard-Smithsonian Center for Astrophysics (CfA), 60 Garden Street, Cambridge, MA 02138; jdrake@cfa.harvard.edu.

${ }^{7}$ Centre for Astrophysics, University of Central Lancashire, Preston PR1 2HE, UK; spseyres@uclan.ac.uk.

${ }^{8}$ Gemini Observatory, 670 North A'ohoku Place, Hilo, HI 96720; tgeballe@gemini.edu.

${ }^{9}$ Joint Astronomy Centre, 660 North A 'ohoku Place, University Park, Hilo, Hawaii 96720; t.kerr@jach.hawaii.edu.

${ }^{10}$ Landessternwarte, Königstuhl, D-69117 Heidelberg, Germany; j.krautter@1sw.uni-heidelberg.de.

${ }^{11}$ The Aerospace Corporation, Mail Stop M2-266, P.O. Box 92957, Los Angeles, CA 90009-2957; david.k.lynch@aero.org, richard.j.rudy@aero.org, ray.russell@aero.org.

${ }^{12}$ School of Earth and Space Exploration, Arizona State University, P.O. Box 871404, Tempe, AZ 85287-1404; sumner.starrfield@asu.edu, ness@susie.la.asu.edu.

${ }^{13}$ Department of Physics and Astronomy, University of Leicester, Leicester LE1 7RH, UK; julo@star.le.ac.uk,kpa@star.le.ac.uk.

${ }^{14}$ Steward Observatory, University of Arizona, 933 North Cherry Avenue, Tucson, AZ 85721; gschwarz@as.arizona.edu.
}

observational campaign. Infrared (IR) observations (Das et al. 2006; Evans et al. 2007a, 2007b) showed evidence for the shock, seen also at radio (Eyres et al. 2007; O'Brien et al. 2006) and X-ray (Bode et al. 2006; Sokoloski et al. 2006; Ness et al. 2007; Osborne et al. 2007) wavelengths, as the ejecta interacted with the RG wind.

Observations of RS Oph obtained with the Spitzer Space Telescope (Werner et al. 2004; Gehrz et al. 2007) during the period from 2006 April 16 through 26 UT $(\simeq 67$ days after outburst) were described by Evans et al. (2007b). We present here further IR observations conducted as part of a long-duration synoptic campaign, obtained later in the outburst with the Spitzer Infrared Spectrometer (IRS; Houck et al. 2004).

\section{OBSERVATIONS}

RS Oph was observed with the Spitzer IRS as part of the Director's Discretionary Time program, Program Identification (PID) 270 on 2006 September 9.83 UT (day 208), 2006 October 10.4 UT (day 246), and 2007 April 19.5 UT (day 367.2). Observations were performed using all IRS modules and the blue peak-up on RS Oph. The spectroscopy consisted of 5 cycles of 14 second ramps in Short-Low mode, 5 cycles of 30 second ramps in both Short-High and Long-Low modes, and 5 cycles of 60 second ramps in Long-High mode. IRS basic calibrated data products (BCDs) were processed with versions 14.4.0 (2006 observations) and 16.1.0 (2007 observation) of the pipeline. The extracted spectra were not defringed. Full details of the data reduction process are given in Evans et al. (2007b) and are not repeated here.

Figure 1 shows, with the same flux density scale and over the wavelength range 6-14 $\mu \mathrm{m}$, the Spitzer spectrum from Evans et al. (2007b) when free-free radiation and fine-structure lines dominated the emission, along with the three new spectra reported in this Letter. In the period covered by the observations reported here there seems to have been little change in the level of the emission: the differences of $\sim 10 \%$ are comparable with the uncertainty in the flux calibration. We conclude that, in 2006 April, emission from the hot gas dominated the dust emission but as the emission from the gas subsided, emission from dust was revealed. The low-resolution Spitzer spectrum of 2006 September 09.83 UT is shown in Figure 2, in which the silicate features at 9.7 and $18 \mu \mathrm{m}$ are clearly visible. 


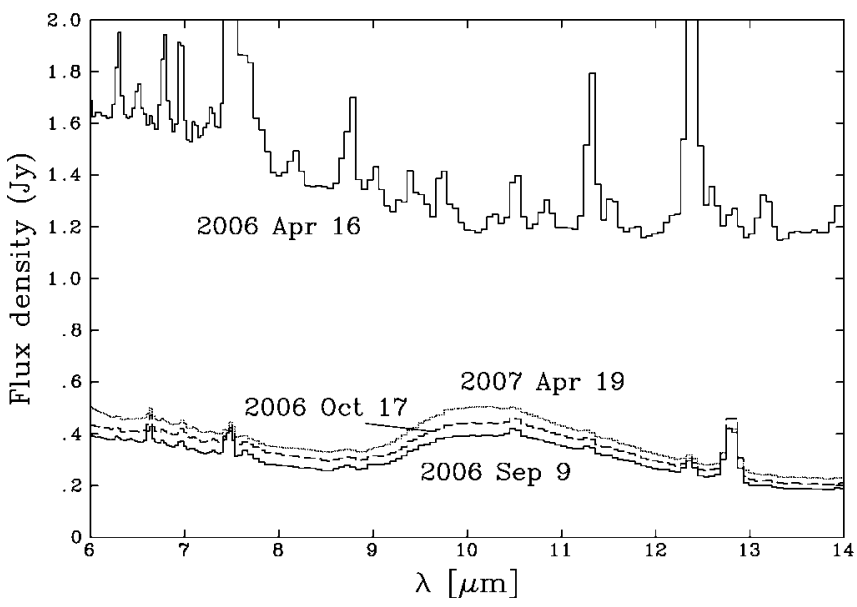

FIG. 1.-Spitzer IRS spectra of RS Oph over the period 2006 April (hot gas dominant) to late 2006 and later (silicate features prominent).

We confine our discussion in this Letter to the silicate features, and defer a discussion of the evolving line and (gas) continuum emission to a later paper.

\section{DISCUSSION}

The formation of silicate dust requires an environment in which $\mathrm{O}>\mathrm{C}$ by number (Whittet 2003). Therefore on the assumption that the silicate dust we see in the Spitzer spectrum arises in the RG wind (see below), we conclude that the RG in the RS Oph system cannot be carbon-rich; this is consistent with the presence of $\mathrm{TiO}$ bands in the optical (Blair et al. 1983), and the presence of deep $\mathrm{O}$ I absorption combined with no carbon absorption in the X-ray range (Ness et al. 2007).

RS Oph is listed as a $12 \mu \mathrm{m}$ source in the IRAS Point Source Catalog, with a flux density (not color corrected) $0.42 \mathrm{Jy}$ and upper limits in the other IRAS bands. To obtain a better estimate of the mid-IR flux densities, we retrieved the original scans from the IRAS database (Assendorp et al. 1995) and used the GIPSY software to measure flux densities from traces through the position of the star. In this way the $12 \mu \mathrm{m}$ value is refined to $0.37 \pm 0.02 \mathrm{Jy}$, and a marginal detection, $0.09 \pm 0.03 \mathrm{Jy}$, is recovered at $25 \mu \mathrm{m}$; RS Oph was not detected at 60 or $100 \mu \mathrm{m}$, to uninterestingly large upper limits. These data, along with the $10 \mu \mathrm{m}$ flux from Geisel et al. (1970), the 2MASS survey ${ }^{15}$ (Skrutskie et al. 2006; data obtained on 1999 April 22), and the Spitzer spectrum from 2006 September, are shown in Figure 3.

Given that the IRAS, 2MASS, and Geisel et al. (1970) data were obtained between eruptions, we are confident that they represent the quiescent state of RS Oph. Moreover, the fact that the Spitzer data are superficially consistent with the interoutburst IR observations (see Fig. 3), and that the dust was present at day 430, suggests that the dust seen by Spitzer is likely present between outbursts (note that Hodge et al. [2004] found no evidence of dust in ISO spectra of RS Oph, but their data only went as far as $9 \mu \mathrm{m}$ ). We have made a preliminary fit using DUSTY (Ivezić \& Elitzur 1997) with a 3600 K blackbody (to represent the RG during quiescence) as input radiation field. Using silicate optical constants from Ossenkopf (1992) we find optical depth $\tau_{V} \simeq 0.1( \pm \sim 10 \%)$, dust temperature at the inner edge of the dust shell $T_{0} \simeq 600 \mathrm{~K}( \pm \sim 100 \mathrm{~K})$, and

\footnotetext{
${ }^{15}$ The 2MASS survey is a joint project of the University of Massachusetts and the Infrared Processing and Analysis Center/California Institute of Technology, funded by NASA and the NSF.
}

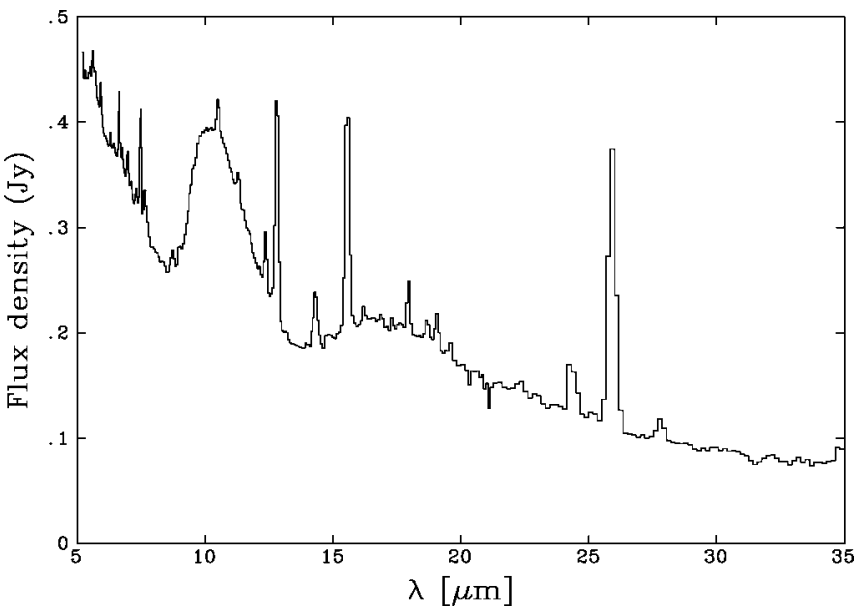

FIG. 2.-Spitzer low-resolution IRS spectrum of RS Oph obtained on 2006 September 9.8 UT. The silicate features at 9.7 and $18 \mu \mathrm{m}$ are clearly visible.

dust shell inner radius $1.5 \times 10^{14} \mathrm{~cm}$. We derive a luminosity $L_{*} \simeq 440 L_{\odot}$, mass-loss rate $\dot{M} \simeq 2.3 \times 10^{-8} M_{\odot} \mathrm{yr}^{-1}$ (typical for these stellar parameters; van Loon et al. 2005) for a dustto-gas mass ratio of 0.005 , and a terminal wind speed $v_{\infty}=$ $8.1 \mathrm{~km} \mathrm{~s}^{-1}$.

While we caution against taking these derived parameters too literally, the "outer" edge of the wind (defined by $v_{\infty} \Delta t$, where $\Delta t$ is the time between the 1985 and 2006 eruptions) is $\simeq 5.4 \times 10^{14} \mathrm{~cm}$. The ejecta from the 2006 eruption, traveling at constant velocity, would have overtaken the outer edge of the wind even at the earliest of our observations ( $t=208$ days). However, the shock, which is decelerating (Bode et al. 2006; Das et al. 2006; Evans et al. 2007a), may not, as yet, have reached the wind edge. Taking $\tau_{v} / \tau_{9.7} \simeq 17.8$ (see Mathis 1998) and silicate opacity from Ossenkopf (1992), we deduce a dust mass of $4.1 \times 10^{-9} M_{\odot}$. Also, the inner radius of the dust shell is rather greater than the binary separation $\left(\simeq 2.2 \times 10^{13} \mathrm{~cm}\right.$, assuming that the mass of the RS Oph system is $\simeq 2 M_{\odot}$ ).

We have determined the full width at half-maximum (FWHM) of the $9.7 \mu \mathrm{m}$ feature by drawing a linear continuum between 8.5 and $12.5 \mu \mathrm{m}$ (see Fig. 4, in which the fluxes at $10 \mu \mathrm{m}$ have been normalized to the 2006 September value). We find that on 2006 September 9 and October 17, the FWHM

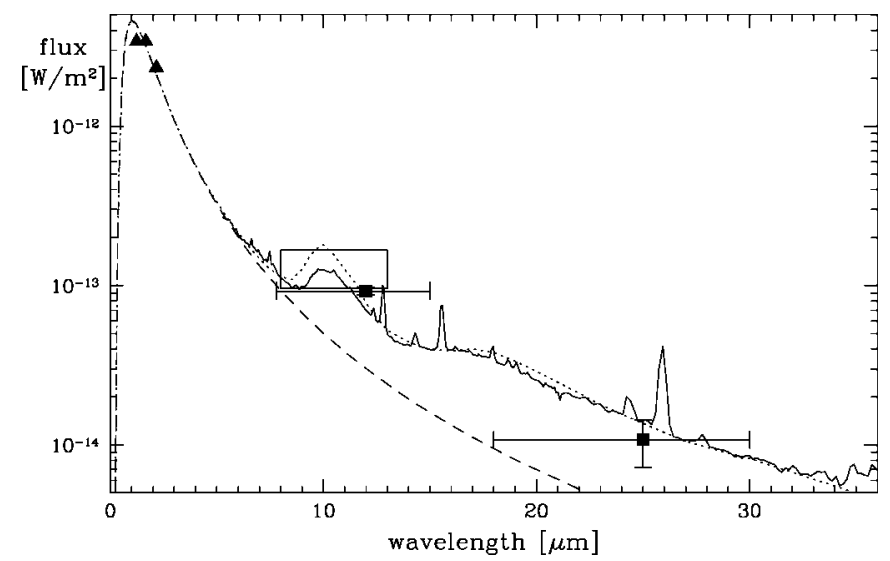

FIG. 3.-Spitzer IRS spectrum from 2006 September 9.8 UT, together with data from 2MASS, IRAS, and Geisel et al. (1970). Triangles, 2MASS; squares with error bars, IRAS; large open square, Geisel et al. (1970). The dashed curve is a $3600 \mathrm{~K}$ blackbody, used as input into DUSTY; the dotted curve is the DUSTY fit. For details see $\S 3$. 


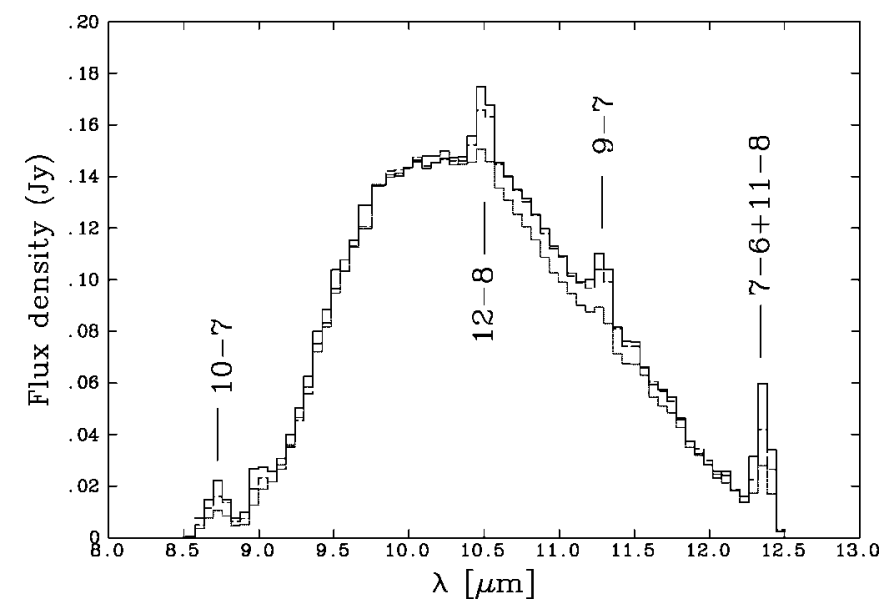

FIG. 4.-Spitzer spectrum of RS Oph for 2006-2007; a linear continuum has been removed from the $8.5-12.5 \mu \mathrm{m}$ region. Principal $\mathrm{H}$ recombination lines $(n-m)$ are identified. Spectra for 2006 September and October are essentially identical; note that the $9.7 \mu \mathrm{m}$ feature for 2007 April (dotted line) is noticeably narrower.

was 2.17 and $2.14 \mu \mathrm{m}$, respectively, with no evidence for change; indeed it is difficult to distinguish the silicate features for these two epochs. By 2007 April 19, however, the FWHM was significantly smaller, $1.99 \mu \mathrm{m}$, perhaps because the dust had experienced some processing (resulting in a change in the dust optical properties) since 2006 September/October. The profile of the $9.7 \mu \mathrm{m}$ feature in RS Oph broadly resembles those of the "Type C" silicate features of AGB stars in the classification by Speck et al. (2000). However, the silicate feature in RS Oph may differ in detail from those of AGB stars for several reasons, such as lack of hot dust in RS Oph, or to grain size, shape, and composition differences. Differences may also arise because the dust in RS Oph is persistently exposed to ultraviolet (UV) radiation from the WD, as well as suffering frequent exposure to hot ionized gas (see below); the latter is known to anneal amorphous silicate (Carrez et al. 2002).

Dust formation in the winds of classical novae during eruption is well documented (Evans \& Rawlings 2008; Gehrz 2008). Indeed, our observations were obtained during an inflection in the visible light curve, where the $V$-band magnitude abruptly decreased by $\sim 0.6 \mathrm{mag}$, recovering shortly thereafter. In classical novae such inflections, accompanied by changes in the IR spectral energy distribution, are associated with dust condensation in the ejecta.

However, in RS Oph we are confident that dust did not form in the ejecta, for two reasons. First, the temperature of the shocked gas $\left(\sim 10^{6} \mathrm{~K}\right.$; Evans et al. 2007a, 2007b) is not conducive to grain formation, which requires dense, relatively cool $(\simeq 2000$ $\mathrm{K})$ gas. Second, the strength of the $18 \mu \mathrm{m}$ feature relative to that of the $9.7 \mu \mathrm{m}$ feature indicates that the dust has been processed. In freshly condensed silicate dust, the $18 \mu \mathrm{m}$ feature is expected to be weak by comparison with the $9.7 \mu \mathrm{m}$ feature (Nuth \& Hecht 1990). Alternatively, the $18 \mu \mathrm{m} / 9.7 \mu \mathrm{m}$ ratio for silicate features in these circumstellar environments could be solely dependent on dust temperature. We have already noted the apparent paucity of hot dust in RS Oph, so the $9.7 \mu \mathrm{m}$ feature will be less strong. In fact our DUSTY fit (Fig. 3) overestimates the 9.7 $\mu \mathrm{m}$ feature strength, even though the model satisfactorily fits the dust continuum as well as the $18 \mu \mathrm{m}$ feature. We conclude that the silicate emission evident in the RS Oph Spitzer spectra is inconsistent with dust formation in the 2006 eruption.

Previous models of the evolution of the remnant of RS Oph following eruption (e.g., Bode \& Kahn 1985; O'Brien et al. 1992) have, for reasons of simplicity, assumed spherical symmetry. Accordingly the ejecta sweep into the RG wind, are shocked, and eventually reach the outer edge of the wind. In this scenario the entire RG wind is completely engulfed by the ejecta, and is replenished only after the eruption has subsided and the RG wind starts anew. As a result any dust condensing in the RG wind would also be engulfed by the shocked gas.

However, the presence of warm silicate dust so soon after the 2006 eruption, and its likely presence between eruptions, may imply that a substantial portion of the RG wind never "sees" the eruption at all. Assuming that the RG fills its Roche lobe, its radius is $\simeq 0.7$ of the binary separation for a mass ratio (secondary/primary) $\simeq 0.4$. It therefore presents a solid angle $\sim 0.3$ steradians to the WD (i.e., it covers $\sim 2.5 \%$ of the WD's sky).

When the RN eruption occurs on the surface of the WD it sweeps past the $\mathrm{RG}$ in $\sim 1.5$ days, potentially arriving at the nominal outer edge of the wind in a few tens of days. This is much less than the orbital period, so $\sim 2.5 \%$ of the wind is shielded by the RG and sees neither the eruption itself nor the expanding ejecta. However, the situation is more complex than this in view of the radial dependence of the wind density, and it is likely that the shocked gas will encroach into the shielded volume. Nevertheless, our estimate of $2.5 \%$ for the fraction of shielded wind is likely of the right order: shielding of the wind by the RG is unlikely to account for the dust we see.

On the other hand, the density of circumstellar material in binaries is greatest in the binary plane (e.g., Spruit \& Taam 2001 and Gehrz et al. (2001) for the case of the massive binary RY Sct), so the RG wind will not be spherically symmetric. Outflow, both of the wind and the 2006 ejecta, will be inhibited in the binary plane and indeed, anisotropies are suggested by HST (Bode et al. 2007) and radio (O'Brien et al. 2006) observations, and IR interferometry (Monnier et al. 2006). We expect a significant amount of dust to survive in the binary plane; such a dense medium would be consistent with the shock deceleration seen in the early Swift (Bode et al. 2006) and IR (Das et al. 2006; Evans et al. 2007a) data.

The higher density material in the binary plane is effectively shielded and may not experience the eruption (which in any case may not have been spherically symmetric); this offers, in part, an explanation for the continued presence of silicate dust in the environment of RS Oph after eruption.

Much of the dust in the RG wind away from the binary plane would not survive the surge of UV radiation emitted at outburst. The temperature of grains at the inner edge of the dust shell would rise by a factor $\left(L_{\mathrm{Edd}} / L_{*}\right)^{1 / 5} \simeq 2.5$ when heated by $\mathrm{UV}$ radiation from a source at the Eddington limit, $L_{\text {Edd }}$, for a Chandrasekhar-mass WD. Thus grains at $600 \mathrm{~K}$ at quiescence would be heated to $\simeq 1500 \mathrm{~K}$, in excess of the nominal evaporation temperature $(\simeq 1300 \mathrm{~K}$; Speck et al. 2000$)$ of silicate dust. Indeed, silicate grains would not survive the eruption out to distance $r_{\text {evap }} \sim 2.2 \times 10^{14} \mathrm{~cm}$ from the WD in outburst. Furthermore, it is likely that a substantial amount of dust may have survived in denser regions swept up in winds from previous outbursts.

Any dust beyond the evaporation distance would survive the UV blast, so we consider whether this dust would survive the passage of the shock. As discussed in earlier papers (Evans et al. 2007a, 2007b), the gas temperature implied by the IR fine structure and coronal lines likely ranges from $\sim 150,000$ to $\sim 900,000 \mathrm{~K}$, with an electron density $\sim 2.2 \times 10^{5} \mathrm{~cm}^{-3}$ in the cooler region; the X-ray data indicate shock temperatures even 
higher than this (Bode et al. 2006). Dust grains in this environment will be sputtered as they are engulfed by the shock. Assuming that the sputtering threshold for silicate grains is $\sim 6$ $\mathrm{eV}$ (Tielens et al. 1994), the timescale for complete erosion of $0.1 \mu \mathrm{m}$ silicate grains in the environment implied by the IR data is $\simeq Y^{-1}$ years, where the yield $Y$ (the number of grain atoms ejected per incident ion) is $\sim 10^{-3}$ (Tielens et al. 1994). As this erosive timescale is $\gg \Delta t$ (the inter-outburst interval) any grains in the RG wind would survive passage of the shock, but might well be processed during the passage of the hot gas.

Our conclusion is consistent with $N$-band interferometric observations conducted on day 3.8, which may have independently seen the dust we report here (Barry et al. 2007). The nearest constructive peak in the interferometer's acceptance pattern is at an angular distance from the central null fringe that depends on wavelength as $\lambda / B$, where $B$ is the length of the projected baseline $(84 \mathrm{~m})$. At the center of the $N$ band, in which Si I $9.407 \mu \mathrm{m}$ and dust are detected, the peak transmission is at angular separation $\simeq 12.5$ mas from the center of the source brightness distribution, equivalent to $\simeq 3 \times 10^{14} \mathrm{~cm}$ at a distance of $1.6 \mathrm{kpc}$. The initial shock velocity would need to have been $\sim 9000 \mathrm{~km} \mathrm{~s}^{-1}$ to reach this distance, traveling at constant velocity, far higher than observed (e.g., Evans et al. 2007a; Bode et al. 2007). Therefore this emission line was not excited by passage of the shock front but by the UV flash from the thermonuclear runaway. This measurement is consistent with our assertion that dust detected by Spitzer was not created by the collision of the ejecta with the RG wind or by subsequent condensation, but was more likely formed during quiescence.

Our continuing Spitzer observations of RS Oph will enable us to examine the effects of irradiation on the dust, and of the re-formation of dust as the circumbinary environment is reestablished by RG wind infill.

\section{CONCLUDING REMARKS}

We have presented further Spitzer IRS observations of RS Oph in the aftermath of its 2006 eruption. Unlike our earlier observation, which showed emission by the hot shocked gas, more recent IR spectra reveal the presence of silicate dust.

The dust we see could not have been formed in the 2006 eruption. It most probably survived the 2006 eruption, because a portion of the RG wind (including the dust) did not see the eruption at all, and because the passing shock failed to destroy any dust that survived the UV blast at the eruption. This conclusion may have major implications for the evolution of the shock, and for the long-term survival of the RG wind between eruptions. We predict that the dust we see around RS Oph will persist until the next eruption.

This work is based on observations made with the Spitzer Space Telescope, which is operated by the Jet Propulsion Laboratory, California Institute of Technology under a contract with NASA; we acknowledge the award of Director's Discretionary Time. L. A. H., C. E. W., and R. D. G. are supported in part by NASA through contracts 1256406, 1215746, and 1267992 issued by JPL/Caltech to the University of Minnesota. T. R. G. is supported by the Gemini Observatory, which is operated by the Association of Universities for Research in Astronomy, Inc., on behalf of the international Gemini partnership of Argentina, Australia, Brazil, Canada, Chile, the United Kingdom, and the United States of America. The work of D. K. L., R. J. R., and R. W. R. is supported by the Aerospace Corporation's Independent Research and Development Program. M. F. B. was supported by a PPARC Senior Fellowship. J.-U. N. was supported by NASA through Chandra Postdoctoral Fellowship grant PF5-60039 awarded by the Chandra X-Ray Center, which is operated by the Smithsonian Astrophysical Observatory for NASA under contract NAS8-03060. J. P. O. and K. L. P. acknowledge support from PPARC. S. S. acknowledges partial support from NSF and NASA grants to Arizona State University.

Facilities: Spitzer (IRS)

\section{REFERENCES}

Assendorp, R., et al. 1995, A\&AS, 110, 395

Barry, R. K., et al. 2007, ApJ, submitted

Blair, W. P., Feibelman, W. A., Michalitsianos, A. G., \& Stencel, R. E. 1983, ApJS, 53, 573

Bode, M. F. 1987, RS Ophiuchi (1985) and the Recurrent Nova Phenomenon (Utrecht: VNU Science Press)

Bode, M. F., \& Kahn, F. 1985, MNRAS, 217, 205

Bode, M. F., et al. 2006, ApJ, 652, 629 2007, ApJ, 665, L63

Carrez, P., Demyk, K., Leroux, H., Cordier, P., Jones, A. P., \& D'Hendecourt, L. 2002, Meteoritics Planet Sci., 37, 1615

Das, R., Banerjee, D. P. K., \& Ashok, N. M. 2006, ApJ, 653, L141

Evans, A., \& Rawlings, J. M. C. 2008, in Classical Novae, ed. M. F. Bode \& A. Evans (2nd ed.; Cambridge: Cambridge Univ. Press), in press

Evans, A., et al. 2007a, MNRAS, 374, L1 2007b, ApJ, 663, L29

Eyres, S. P. S., et al. 2007, MNRAS, submitted

Fekel, F. C., Joyce, R. R., Hinkle, K. H., \& Skrutsie, M. F. 2000, AJ, 119, 1375

Gehrz, R. D. 2008, in Classical Novae, ed. M. F. Bode \& A. Evans (2nd ed.; Cambridge: Cambridge Univ. Press), in press

Gehrz, R. D., Smith, N., Jones, B., Puetter, R., \& Yahil, A. 2001, ApJ, 559, 395

Gehrz, R. D., et al. 2007, Rev. Sci. Instrum., 78, 01130

Geisel, S. L., Kleinmann, D. E., \& Low, F. J. 1970, ApJ, 161, L101

Hodge, T. M., Kraemer, K. E., Price, S. P., \& Walker, H. J. 2004, ApJS, 151, 299

Houck J. R., et al. 2004, ApJS, 154, 18

Ivezić Ž., \& Elitzur M. 1997, MNRAS, 287, 799
Mathis, J. S. 1998, ApJ, 497, 824

Monnier, J. D., et al. 2006, ApJ, 647, L127

Narumi, H., Hirosawa, K., Kanai, K., Renz, W., Pereira, A., Nakano, S. Nakamura, Y., \& Pojmanski, G. 2006, IAU Circ., 8671, 2

Ness, J.-W., et al. 2007, ApJ, 665, 1334

Nuth, J. A., III, \& Hecht, J. H. 1990, Ap\&SS, 163, 79

O'Brien, T. J., Bode, M. F., \& Kahn, F. D. 1992, MNRAS, 255, 683

O'Brien, T. J., et al. 2006, Nature, 442, 279

Osborne, J. P., et al. 2007, ApJ, submitted

Ossenkopf, V., Henning, T., \& Mathis, J. S. 1992, A\&A, 261, 567

Skrutskie, M. F., et al. 2006, AJ, 131, 1163

Sokoloski, J., Luna, G. J. M., Mukai, K., \& Kenyon, S. J. 2006, Nature, 442, 276

Speck, A., Barlow, M. J., Sylvester, R. J., \& Hofmeister, A. M. 2000, A\&AS, 146,437

Spruit, H. C., \& Taam, R. E. 2001, ApJ, 548, 900

Starrfield, S., Sparks, W. M., \& Shaviv, G. 1988, ApJ, 325, L35

Tielens, A. G. G. M., McKee, C. F., Seab, C. G., \& Hollenbach, D. J. 1994, ApJ, 431, 321

van Loon, J. T., Cioni, M.-R., L., Zijlstra, A. A., \& Loup, C. 2005, A\&A, 438, 273

Wallerstein, G. 2008, in ASP Conf. Ser., RS Ophiuchi (2006) and the Recurrent Nova Phenomenon, ed. A. Evans, M. F. Bode, \& T. J. O'Brien (San Francisco: ASP), in press

Werner, M. W., et al. 2004, ApJS, 154, 1

Whittet, D. C. B. 2003, Dust in the Galactic Environment (2nd ed.; Bristol: IoP Publishing) 